4. Mehta RH, Manfredini R, Hassan F, Sechtem U, Bossone E, Oh JK, et al. Chronobiological patterns of acute aortic dissections. Circulation. 2002; 106:1110-5.
5. Sega R, Cesana G, Bombelli M, Grassi G, Stella ML, Zanchetti A, et al. Seasonal variations in home and ambulatory blood pressure in the PAMELA population. Pressione Arteriose Monitorate e Loro Associazioni. J Hypertens. 1998;16:1585-92.

\title{
Multiple thoracic aortic aneurysms after mediastinitis in an infant after repair of coarctation of the aorta
}

\author{
Laurie E. Profitlich, MD, ${ }^{*}$ Constance G. Weismann, MD, ${ }^{*}$ Shubika Srivastava, MD, Bruce D. Gelb, MD, \\ Kahn Nguyen, MD, and Umesh Joashi, MD, New York, NY
}

Supplemental material is available online.

\section{$\mathrm{W}$}

Clinical Summary e describe the case of a 3.1-kg term infant with critical aortic coarctation transferred to our institution on the second day after birth. There was no risk factor for infection in the perinatal or birth history. An umbilical arterial catheter (UAC) and venous catheters were placed and a prostaglandin $\mathrm{E}_{1}$ infusion was initiated. Intermittent low-grade fever on days 4 and 5 after birth was attributed to the prostaglandin therapy.

On day 6 , the patient was taken to the operating room for a resection and end-to-end anastomosis of a discrete coarctation through a left lateral thoracotomy. On entering the chest, we encountered purulent fluid in the mediastinum, and the aorta was adherent to the surrounding tissue, precluding mobilization. A subclavian flap repair was performed. The mediastinum was cultured and irrigated. The patient was transferred to the cardiac intensive care unit where vancomycin, cefepime, and gentamicin were started empirically. The UAC was removed. Osteomyelitis then developed. Blood and wound cultures persistently grew multi-drug resistant Staphylococcus aureus. The cultures finally became sterile on postoperative day 9 after modification of antibiotics (daptomycin, rifampin, and linezolid).

A chest computed tomogram (CT) performed on postoperative day 8 (Figure 1, A) showed multiple lung abscesses and a large, ring-enhancing mycotic aneurysm of the descending thoracic aorta

From the Department of Pediatric Cardiology, Mount Sinai Medical Center, New York, NY.

* Drs Profitlich and Weismann are joint first authors.

Received for publication Sept 7, 2007; accepted for publication Sept 20, 2007.

Address for reprints: Constance Weismann, MD, One Gustave L. Levy Place, New York, NY, 10029. (E-mail: constance.weismann@mssm.edu and laurie. profitlich@mssm.edu).

J Thorac Cardiovasc Surg 2008;135:444-5

$0022-5223 / \$ 34.00$

Copyright $\odot 2008$ by The American Association for Thoracic Surgery doi:10.1016/j.jtcvs.2007.09.024 measuring $1.1 \times 1.2 \mathrm{~cm}$ in continuity with a left-sided empyema. The nonaneurysmal aorta measured $0.6 \mathrm{~cm}$ in diameter. Despite antibiotic therapy and careful blood pressure control, a repeat chest CT done on postoperative day 13 (Figure $1, B$ ) showed a significant increase in the size of the aneurysm to $2.4 \times 1.5 \mathrm{~cm}$. Interval echocardiograms confirmed aneurysmal enlargement (Figure 2, $A$ and $B$ ). Also, a proximal aneurysm was detected just distal to the anastomosis of the subclavian flap.

A chest CT on postoperative day 28 (Figure 1, C) measured the distal aneurysm at $2.3 \times 3.9 \times 2.8 \mathrm{~cm}$ and the proximal aneurysm at $1.4 \times 2.2 \times 1.8 \mathrm{~cm}$. Owing to the rapidly increasing size and risk of rupture, an aortic bypass graft with exclusion of the entire thoracic descending aorta was performed on postoperative day 30. Via a median sternotomy, an 8-mm Hemashield graft (Boston Scientific, Natick, Mass) was anastomosed in an end-to-side fashion to the ascending aorta and to the descending aorta superior to the celiac trunk.

Eight days later, the patient hemorrhaged acutely from the mediastinal chest tube. A repeat chest CT (Figure 1,D) showed possible retrograde filling of the distal aortic aneurysm from collaterals supplied by intercostal arteries. Subsequent cardiac catheterization and angiography showed a widely patent aortic graft and confirmed retrograde filling of the aneurysm (Figure E1, $A$ and $B$ ).

A repeat chest CT 17 days after graft placement demonstrated continued filling of the thoracic aneurysm via intercostal arteries. The patient then underwent debulking of the aneurysm with removal of a large organized thrombus at that time. An actively bleeding intercostal artery was oversewn.

The patient recovered from the procedure well and was discharged home 8 days later receiving oral linezolid. At most recent follow-up, the patient was 8 months old and was enjoying normal growth and development. The graft was without anastomotic obstruction according to an echocardiographic assessment. Assuming normal somatic growth, we anticipate further surgical intervention will be necessary at the age of 5 or 6 years.

\section{Discussion}

Development of multiple aortic aneurysms in neonates associated with UAC placement is widely described in the literature. ${ }^{1}$ However, aortic aneurysm occurring secondary to mediastinal infection is rare.

UACs are placed in $10.8 \%$ to $64.4 \%$ of neonatal intensive care unit admissions. The incidence of complications is directly related to the duration of catheter use, ${ }^{2}$ and infection is strongly associated 

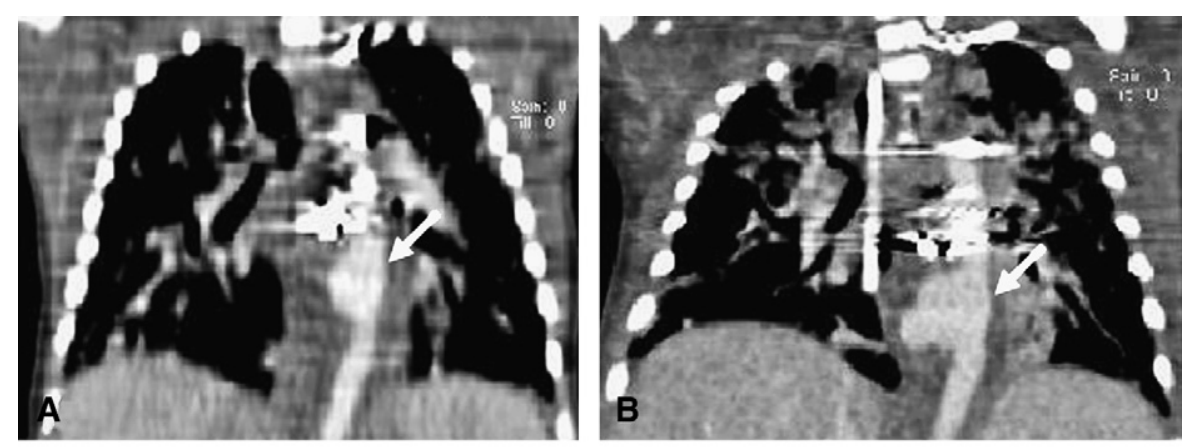

Figure 1. A to $C$, Serial CT scans on postoperative days 8 (A), 13 (B), and 28 (C) showing progression of descending aortic aneurysms (white arrows). D, Retrograde filling of the distal aortic aneurysm (white arrowheads) via collaterals supplied by intercostal arteries 8 days after aortic bypass graft.
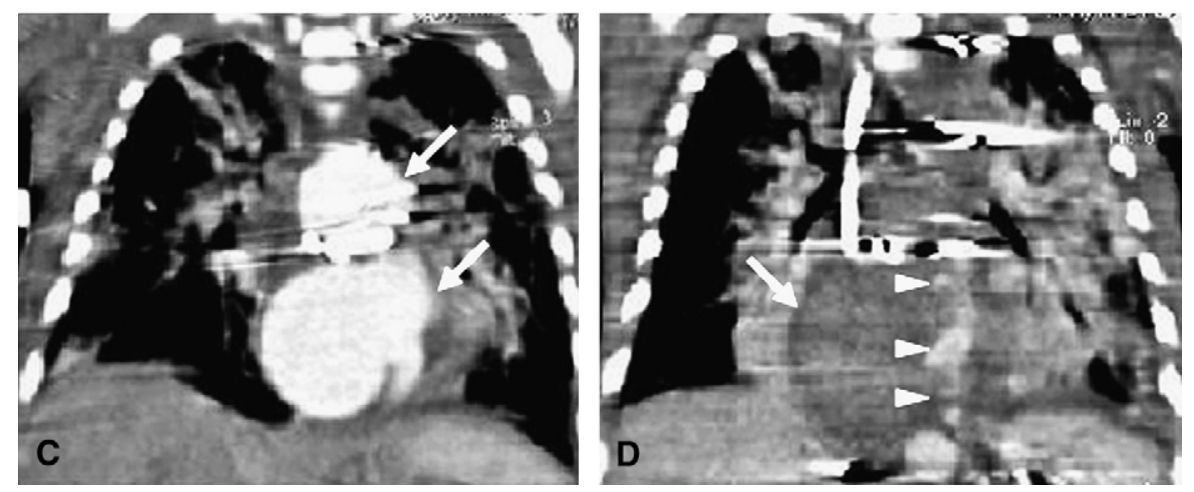

Figure 2. Serial 2-dimensional echocardio-
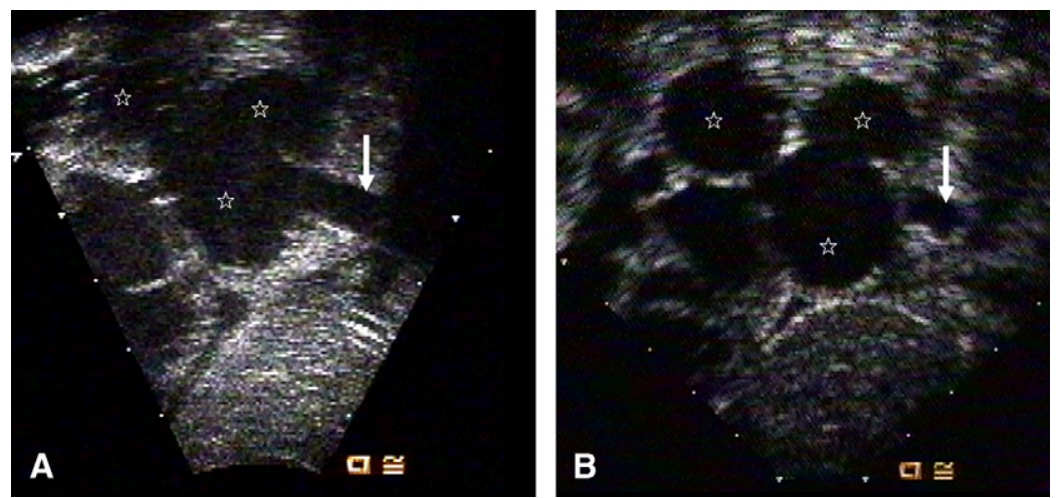
graphic images on postoperative days 20 (A) and 27 (B) showing progression of descending aortic aneurysms (white stars) and part of the unaffected descending aorta (white arrows).

with aneurysm formation. ${ }^{2,3}$ Mechanical trauma from the catheter causes endothelial damage and thrombosis, ${ }^{4}$ increasing vessel wall inflammation and weakness. Catheter tip position is associated with the site of aneurysm formation. ${ }^{3}$ In our patient, the proximal aneurysm developed at the site of the surgical repair. Aneurysms may occur in the abdominal aorta (43\%), thoracic aorta (30\%), iliac artery $(15 \%)$, and thoracoabdominal aorta $(12 \%)$, with the majority being saccular. ${ }^{2}$

The finding of pus in the mediastinum of our patient at the time of surgery suggests a primary extravascular infection. A review of the literature revealed only 2 case reports describing the development of aortic aneurysms secondary to mediastinal infection. ${ }^{5}$ Although there was no clear history of traumatic intubation or difficulty with nasogastric tube placement, we speculate that traumatic esophageal perforation led to bacterial seeding in the mediastinum. It is our assertion that the development of multiple thoracic aortic aneurysms in our patient was a consequence of mediastinitis.

\section{References}

1. Kirpekar M, Augenstein H, Abiri M. Sequential development of multiple aortic aneurysms in a neonate post umbilical arterial catheter insertion. Pediatr Radiol. 1989;19:452-3.

2. Mendeloff J, Stallion A, Hutton M, Goldstone J. Aortic aneurysm resulting from umbilical artery catheterization: case report, literature review, and management algorithm. $J$ Vasc Surg. 2001;33:419-24.

3. Drucker DE, Greenfield LJ, Ehrlich F, Salzberg AM. Aorto-iliac aneurysms following umbilical artery catheterization. J Pediatr Surg. 1986; 21:725-30.

4. Chidi CC, King DR, Boles ET Jr. An ultrastructural study of the intimal injury induced by an indwelling umbilical artery catheter. J Pediatr Surg. 1983;18:109-15.

5. Blickenstaff DE, Nice CM. Aortic aneurusm secondary to mediastinal abscess. Report of two cases. Dis Chest. 1965;48:534-8. 

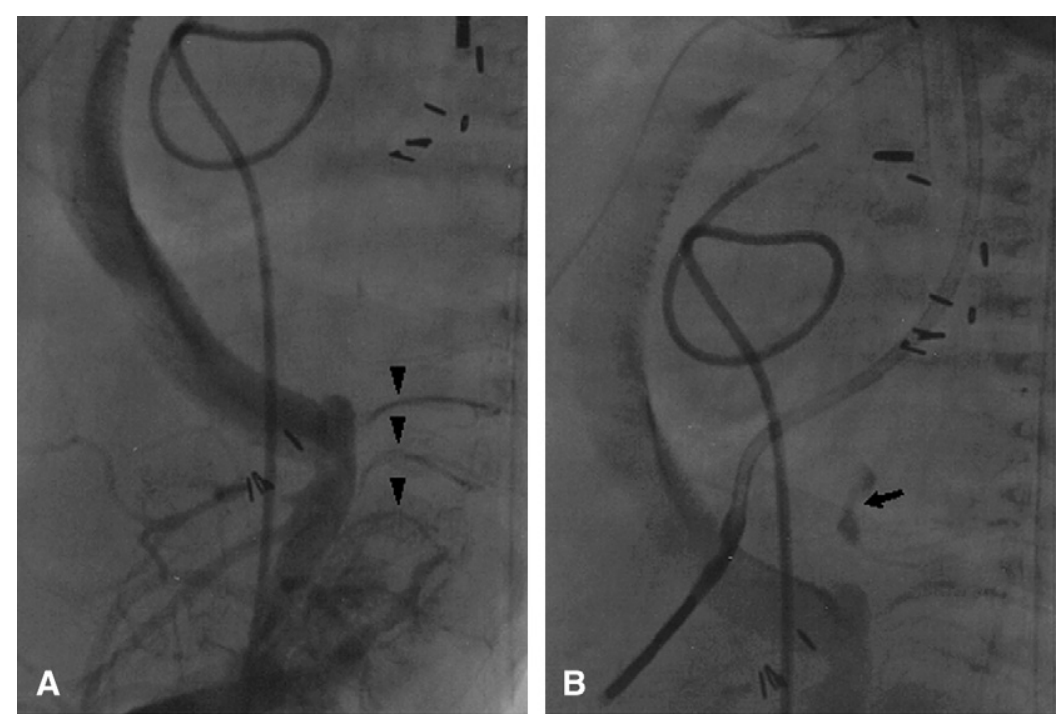

Figure E1. Angiogram of contrast injection into the ascending aorta. $A$, There is contrast in the aortic bypass graft, the abdominal aorta, and in its collateral vessels (black arrowheads). B, Retrograde filling of the clipped off descending aortic aneurysm (black arrow) via collaterals. 